NIST Special Publication 1133

DOI: http://dx.doi.org/10.6028/NIST.SP.1133

\title{
Fourth NIST Workshop on Carbon Nanotubes: Chirality Measurements
}

Summary, Program and Abstracts

September 23 - 24, 2010

Gaithersburg, Maryland, USA

Organizing Committee

Stephen Freiman

Jeffrey A. Fagan

Stephanie Hooker

Kalman B. Migler

Angela R. Hight Walker

Ming Zheng

MATERIAL MEASUREMENT LABORATORY

Polymers Division

NLT

National Institute of Standards and Technology

U.S. Department of Commerce

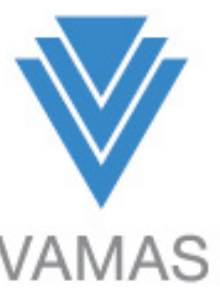


NIST Special Publication 1133

\section{Fourth NIST Workshop on Carbon Nanotubes: \\ Chirality Measurements}

\section{Summary, Program and Abstracts}

September 23-24, 2010

Polymers Division

Material Measurement Laboratory

http://dx.doi.org/10.6028/NIST.SP.1133

January 2013

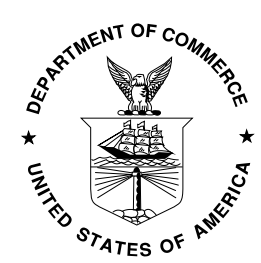

U.S. Department of Commerce Rebecca M. Blank, Acting Secretary

National Institute of Standards and Technology 
Certain commercial entities, equipment, or materials may be identified in this document in order to describe an experimental procedure or concept adequately. Such identification is not intended to imply recommendation or endorsement by the National Institute of Standards and Technology, nor is it intended to imply that the entities, materials, or equipment are necessarily the best available for the purpose.

National Institute of Standards and Technology Special Publication 1133

Natl. Inst. Stand. Technol. Spec. Publ. 1133, 28 pages (January 2013) 


\section{Table of Contents}

Summary

Agenda

Presentation Abstracts

Poster Abstracts

\section{Organizing Committee}

Stephen Freiman

Jeffrey A. Fagan

Stephanie Hooker

Kalman B. Migler

Angela R. Hight Walker

Ming Zheng

Hosted by the National Institute of Standards and Technology 



\section{Summary}

On September $23^{\text {rd }}$ and $24^{\text {th }}$, 2010, the National Institute of Standards and Technology (NIST) hosted the fourth in a series of workshops addressing measurement needs for single wall carbon nanotubes (SWCNTs). Attendees representing an international community, including members of industry, academia, government laboratories, and regulatory agencies, participated in the workshop. The primary focus of this workshop was on SWCNT chirality population measurements and chirality-dependent properties.

The workshop consisted of three sessions. The first morning was devoted to presentations and discussion of an inter-laboratory study on various methods for determination of the chirality distribution of SWCNTs, which was conducted under the auspices of the Versailles Project on Advanced Measurements and Standards (VAMAS). Such measurements are critical for specifying the properties that can be expected from and the purity of nanotubes for commerce. These methods included tipenhanced Raman spectroscopy, fluorimetric analysis, Raman spectroscopy, and transmission electron microscopy (TEM). The material used in the inter-laboratory study was the Long fraction of a NIST reference material that will be publicly available for all purchasers to compare against (RM 8281, length-separated SWCNT populations). So far, 12 of the laboratories, representing seven countries, participating in this round robin have submitted results.

The early results of the inter-laboratory study show dramatically how different techniques and their level of difficulty affect the measured population distribution. The advantages and disadvantages of various techniques imply that continued caution is needed in interpreting population data. The results of the study will be published and used to enable techniques for the absolute determination of the SWCNT chiral vector distribution in a dispersed population.

The second session focused on techniques for chirality separation and selective synthesis. Most of these presentations dealt with advances in the methods for separating nanotube populations. Highlights were detailed discussion of three different techniques: density gradient ultracentrifugation (DGU), agarose gel column chromatography, and ion-exchange chromatography using sequence-selective DNA wrapping of the nanotubes. Specifically, Mark Hersam described advances in density gradient centrifugation that could be used to create samples of $98 \%$ to $99 \%$ pure SWCNT populations. He additionally discussed a number of new nanotube-based devices that could result from this separation technique. Hiromichi Kataura presented a multi-stage chirality separation via a gel-filtration method that enables sequential isolation of all the small-diameter semiconducting chiralities in a HiPco sample. Xiaomin Tu presented the technique of sequence-selective DNA-based chirality separation, and new work supporting the DNA structure - selectivity hypothesis. As presented by Nathan Yoder of Nanointegris corp., the cost of separated SWCNTs, and ultimately the cost of any application derived from these nanotubes, is critically dependent on the ability of the separation technologies to produce them in the needed chiralities and sizes in sufficiently large quantities.

Separately, R. Mohan Sankaran and Sivaram Arepalli both talked about methods for influencing the distribution of synthesized nanotubes. These included control of the catalyst size and synthesis conditions. Results presented showed that these variables can shift the synthesized population of nanotubes, and may lessen the need for post-production separation techniques.

The final session was devoted to applications that are enabled by chirality-separated SWCNT populations. These included field-effect transistors, thin-film solar devices (Jeffrey Blackburn and Michael Arnold), and nano-therapeutics and combined targeting / bioimaging agents (Hongjie Die and 
Shawn Chen). It was also noted that the purification of single nanotube species is allowing the study of previously intractable photophysical measurements (Stephen Doorn).

The talks on the second day also acknowledged a reality brought up in the earlier session: Although there have been significant advances in separation technologies for large-scale production of different nanotube populations, significant efforts toward rapid quality control are still necessary to commercialize advanced applications. Similarly, there was discussion about the need for improved capabilities for specification of the chiral purity in samples for various electronic applications. There was a consensus that ability to specify the semiconducting purity to several decimal places is needed, with five 9's (99.999 \%) or more being desirable. However, the difficulties that have been encountered in this specification are not just in the removal of intransigent impurity species, but also the missing ability to rapidly screen materials and identify contaminating species. A lack of tools was specifically identified for separation of chiralities with diameters larger than $1 \mathrm{~nm}$.

\section{Conclusions}

Although only a few short years have passed since the previous nanotube workshop at NIST, tremendous progress has been made in the separation and utilization of different SWCNT chiralities and populations. Despite the economic climate, industrial production and use of purified and controlled nanotube populations has emerged to bridge the gap between material science and applied products.

The participants in this workshop were uniformly enthusiastic about nanostructured carbon materials and share the opinion that chiral separation techniques and measurement procedures are important areas for continuing work. Although participants were excited about the progress in isolation of specific nanotube functionalities, the continuing obstacle to the development of many nanotube-based electronics and the highest technological applications is the limited availability of single-chirality SWCNTs in sufficient volumes.

The exciting progress of nanotube science and technologies in the last few years has not eliminated the need for advanced measurement science, but is pushing it in new directions for more advanced control and rapid screening, that will result in new capabilities and applications of nanotube materials. 
Agenda

\section{Thursday, September 23, 2010}

8:15 am Transportation from Hilton to NIST

8:30 Registration and Coffee

9:00 Introduction: Kalman Migler

9:15 NIST Welcome: Katharine Gebbie, Director, Physics Laboratory

\section{Session 1: Interlaboratory Comparison of Chirality Measurements} Session Chair, Jeffrey Fagan

9:30 Jeffrey Fagan, NIST An Overview and Goals for Improving Chiral Vector Distribution Determination through VAMAS TWA 34

9:55 Deb Roy, (NPL, England)

Prospects and Challenges in Characterising SWCNTs Using Tip-Enhanced Raman Spectroscopy

10:20 Break

10:45 Bruce Weisman (Rice University)

Progress in Optical Spectroscopic Analysis of Single-walled Carbon Nanotubes

11:10 Erlon Ferreira (INMETRO)

Resonance Raman RBM Intensity Analysis of the NIST sample RM 8281 Long Fraction

11:35 Hua Jiang (Aalto University, Finland)

Electron Microscopy Measurement of the Chirality Distribution in a Single-Walled

Carbon Nanotube Sample for the VAMAS TWA-34 Project of NIST

12:00 pm Discussion of results and future directions

12:30 Lunch (NIST Cafeteria)

Session 2: $\quad$ Chirality Separation and Selective Synthesis Session Chair, Angela Hight-Walker

1:30 Keynote Lecture

Ado Jorio de Vasconcelos (Universidade Federal de Minas Gerais, Brazil)

The Application of Raman spectroscopy to the Metrology of Carbon Nanotubes

2:10 Mark Hersam (Northwestern Univ.)

Fundamentals and Applications of Monodisperse Carbon-Based Nanomaterials

2:35 Hiromichi Kataura (AIST, Japan)

Chirality Separation by Gel-Filtration Method

3:00 Break

3:25 R. Mohan Sankaran (Case Western Reserve Univ.)

The Role of Composition-Dependent Catalyst Structure on Chirality Distributions of AsGrown SWCNTS 
Fourth NIST Workshop on Carbon Nanotubes: Control and Measurement of Chirality 
3:50 Nathan Yoder (Nanointegris)

Large-Scale Production of Monodisperse Carbon Nanomaterials at NanoIntegris

4:15 Helen Tu (NIST)

Understanding the DNA-Based Carbon Nanotube Chirality Separation

4:40 Discussion

5:00 Poster Session + Reception in Hall of Flags, NIST

6:30 Transportation to Hilton

Friday, September 24, 2010

8:15 am Transportation to NIST

8:30 Coffee

Session 3: $\quad$ Measurements and Applications of Chirality Separated SWCNTs Session Chair, Ming Zheng

9:00 Hongjie Dai (Stanford Univ.)

Carbon Nanotubes for Nanoelectronics and Nanobiotechnolgy

9:25 Jeffrey Blackburn (NREL)

Performance of Type-separated Single-wall Carbon Nanotubes in Energy Conversion Schemes: Charge Transfer and Transport

9:50 Stephen Doorn (LANL)

Resonance Raman Studies of Chirality-Enriched Single-Walled Carbon Nanotubes

10:15 Break

10:40 Shawn Chen (NIH/NIBIB)

Single-Walled Carbon Nanotubes for Cancer Imaging and Therapy

11:05 Michael Arnold (Univ. of Wisconsin)

Exploiting Chirality-Controlled Carbon Nanotubes to Directly Harvest Light and Energy via a Photovoltaic Effect on a Macroscopic Scale

11:30 Sivaram Arepalli (Sungkyunkwan University, Korea)

Growth and Separation of Chirality Controlled SWCNTs

12:00 pm Working Lunch in AML (Future plans, output, next workshop, etc.)

1:00 Adjourn

NIST Laboratory Tours: Please signup if interested 


\title{
Presentation Abstracts
}

\section{AN OVERVIEW AND GOALS FOR IMPROVING CHIRAL VECTOR DISTRIBUTION DETERMINATION THROUGH VAMAS TWA 34}

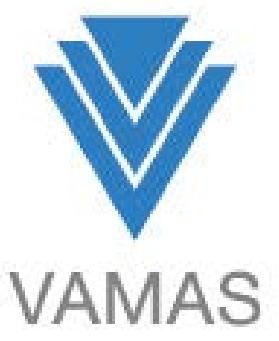

\author{
Jeffrey A. Fagan \\ National Institute of Standards and Technology, Polymers Division, Gaithersburg, \\ MD 20899 \\ jeffrey.fagan@nist.gov
}

The ability to rapidly and easily determine the chiral vector distribution within a nanotube population is a key measurement need for carbon nanotube processing and applications. Under the auspices of technical working area (TWA) 34, Nanoparticle Populations, of the Versailles Project on Advanced Materials and Standards (VAMAS) a round robin measurement of the chirality distribution of a single, purified, single-wall carbon nanotube (SWCNT) source material is being conducted. The goal of this round robin is to provide a basis for comparison between different measurement methodologies, measurement types and laboratories implementations, of the chirality specification for a given sample. Preliminary results from multiple orthogonal techniques for measuring the chiral vector distribution will be discussed in this session. An overview of the goals for discussion of these results, and the incorporation of the data to standards activities will be presented.

\section{Prospects AND Challenges in Characterising SWCNTS USING TiP-Enhanced RAMAN SPECTROSCOPY}

\author{
Deb Roy \\ National Physical Laboratory, Hampton Road, Teddington TW11 OLW, UK \\ debdulal.roy@npl.co.uk
}

Tip-enhanced Raman spectroscopy (TERS) is a novel technique having the high spatial resolution of an atomic force microscopy (AFM) and chemical specificity of Raman spectroscopy to achieve high resolution chemical and structural information (Roy, Wang and Welland 2006). Enhancement factor and spatial resolution are two of the most important metrological parameters that define the quality of a TERS measurement (Roy, Wang and Williams 2009). The technique has been used to image single SWCNTs as well as characterise their structures. First part of the talk will briefly describe the technique, its application on studying isolated SWCNTs, perceived metrology challenges to obtained reproducible measurements. The second part will address the steps towards chiral characterisation of isolated tubes by combining AFM and Raman spectroscopic investigations. Measurements obtained on the VAMAS sample from NIST will also be presented.

\section{References:}

Roy, D., J. Wang and M. Welland (2006). "Nanoscale Imaging of Carbon Nanotubes using Tip Enhanced Raman Spectroscopy in Reflection Mode." Faraday Discussions 132: 215-225.

Roy, D., J. Wang and C. Williams (2009). "Novel methodology for estimating the enhancement factor for tipenhanced Raman spectroscopy." Journal of Applied Physics 105(1). 


\title{
Progress in Optical Spectroscopic Analysis of Single-Walled Carbon Nanotubes
}

\author{
R. Bruce Weisman \\ Rice University, Houston, Texas USA
}

Recent progress will be discussed in the development of optical spectroscopies to analyze bulk samples of single-walled carbon nanotubes (SWCNTs). Since the 2002 discovery and spectral assignment of near-infrared (NIR) fluorescence from semiconducting SWCNTs, optical absorption and emission methods have emerged as powerful tools for detailed nanotube structural analysis. Although these methods provide secure qualitative $(n, m)$ identification, the quantitative determination of $(n, m)$ distributions requires knowledge of structure-dependent absorptivities and/or emissive quantum yields. Calibrated photometric measurements on selected individual nanotubes have now provided absolute intrinsic values of fluorescence action cross sections for 31 different semiconducting $(n, m)$ species. Structural patterns in these values have been deduced and used to extrapolate fluorescence action cross sections for additional species. This set of empirically-based sensitivity factors now allows quantitative fluorimetric determination of semiconducting $(n, m)$ distributions in bulk SWCNT samples.

Advances have also been made in specialized optical instrumentation for efficient SWCNT analysis. Highly sensitive multi-mode spectrometers are now available with four discrete laser wavelengths for exciting NIR fluorescence, absorption capability in the visible and NIR regions, and single-wavelength Raman spectrometry. Updated data analysis software deduces $(n, m)$ distributions (using the new sensitivity factors) and automatically compares spectrally integrated fluorescence emission to sample absorbance at the excitation wavelength to give a relative measure of fluorescence efficiency. This efficiency parameter provides insights into sample dispersion and condition. By mounting the sample holder on a computer-controlled vertical translation stage, the multi-mode spectrometer can perform in situ spectral mapping of undisturbed sample tubes used for density gradient ultracentrifugation (DGU) of SWCNT samples.

Our spectral mapping method has allowed the development of DGU with nonlinear gradients that are tailored for improved $(n, m)$ sorting. HiPco samples have been processed to give fractions enriched in ten different $(n, m)$ species and also for separation of seven pairs of SWCNT enantiomers. The availability of such sorted and purified SWCNT samples is a key step in enabling more detailed spectroscopic and photophysical studies. One new application is an investigation of the broad backgrounds seen in SWCNT absorption spectra. Using a combination of sorted and unsorted samples, we identify and in some cases quantify background contributions from amorphous carbon, chemical functionalization, spectral congestion, aggregation, sonication-induced damage, and metallic species. These findings should enhance the value of optical spectroscopic analysis of SWCNT samples.

\section{RESONANCE RAMAN RBM INTENSITY ANALYSIS OF THE NIST SAMPLE RM 8281 LONG FRACTION}

\author{
Erlon Ferreira \\ Brazilian National Institute of Metrology
}

Here we present a methodology and the results of an intensity analysis of the Resonance Raman radial breathing mode (RBM) signal of the single wall carbon nanotube sample in order to determine its diameter and chirality distribution. The method consists of correcting the obtained RBM signal by the Resonance Raman cross-section, as calibrated by the "super-growth" water assisted chemical vapor deposition (CVD) sample. 


\title{
ELECTRON MiCROSCOPY MEASUREMENT OF THE CHIRALITY DisTRIBUTION IN A SiNGLE-WALLED CARBon NANOTUbE SAMPLE FOR THE VAMAS TWA-34 PROJECT OF NIST
}

\author{
Hua Jiang \\ Department of Applied Physics, Aalto University, Aalto Finland
}

Presented in this contribution are results of single-walled carbon nanotube (SWCNT) chirality distribution determination from a round robin sample for the Versailles Project on Advanced Materials and Standards Technical Working Area 34 (VAMAS TWA 34), which is initiated to provide correlation between the absolute distribution of chiralities present in the sample and the more easily measured properties of the sample.

The measurements were carried out by using electron diffraction and high-resolution imaging techniques in an aberration-corrected transmission electron microscope (TEM). The length sorted SWCNT sample was received in an aqueous $10 \mathrm{~g} / \mathrm{L}$ sodium deoxycholate solution. For TEM observation, a drop of the original SWCNT solution was casted onto a standard TEM grid and dried naturally. Then, the grid is washed carefully using acetone, SWCNTTGF, ethanol, and D.I. water to wash the surfactant away from the surface of the nanotubes.

A large number of electron diffraction patterns (EDPs) and high-resolution TEM images were taken from the sample. From the EDP of an individual SWCNT, the absolute determination of the chiral vector of the nanotube can be obtained. From the EDP of a bundle of SWCNTs, chiral angle distribution in the bundle can be analyzed. For those SWCNTs which are too short, or not isolated enough for decent electron diffraction analysis, aberration-corrected high-resolution TEM imaging technique is applied to accurately measure the diameter distribution in the SWCNT population. At last, a combined statistic result is provided which represents a corner of the round-robin measurements for chirality distribution determination.

\section{The Application of RAman Spectroscopy to the Metrology of CaRbon Nanotubes}

Ado Jorio

\section{Universidade Federal de Minas Gerais}

This talk reviews 10 years of research on the Raman spectroscopy of carbon nanotubes, with focus on the metrological aspects. The $(\mathrm{n}, \mathrm{m})$ assignment, including population analysis will be addressed, with attention to sample comparison and environmental effects. The role of standards is discussed as means to reach deep theoretical understanding of the environmental and $(n, m)$ dependence of the optical properties in general, which can also be applied to photoluminescence and optical absorption characterization. If possible I will discuss also how graphene is helping us to understand the defectinduced Raman features in sp2 nanocarbons. 


\title{
Fundamentals and Applications of Monodisperse Carbon-Based NanOMaterials
}

\author{
Mark C. Hersam \\ Northwestern University
}

\section{http://www.hersam-group.northwestern.edu/}

Carbon-based nanomaterials have attracted significant attention due to their potential to enable and/or improve applications such as transistors, transparent conductors, solar cells, batteries, water purification systems, infrastructure materials, drug delivery, and biosensors [1,2]. This talk will delineate chemical strategies for tuning and enhancing the properties of these promising nanomaterials. For example, we have developed [3,4] and commercialized [5] a scalable technique for sorting single-walled carbon nanotubes (SWCNTs) by their physical and electronic structure using density gradient ultracentrifugation (DGU). The resulting monodisperse SWCNTs possess unprecedented uniformity in their electronic and optical properties, which enables the fabrication of high performance thin film fieldeffect transistors [6-8], optoelectronic devices [6,9], and transparent conductors [10]. The DGU technique also enables multi-walled carbon nanotubes to be sorted by the number of walls [11], and solution phase graphene to be sorted by thickness [12,13], thus expanding the suite of monodisperse carbon-based nanomaterials. By recently extending our DGU efforts to SWCNTs and graphene dispersed in biocompatible polymers (e.g., DNA, poloxamers, etc.), new opportunities have emerged in biomedical applications $[14,15]$. Ultimately, the ability to control structure and surface chemistry with sub-nanometer precision enables optimized properties for a diverse range of technologies that employ carbon-based nanomaterials.

[1] M. C. Hersam, Nature Nanotechnology, 3, 387 (2008).

[2] J. Liu and M. C. Hersam, MRS Bulletin, 35, 315 (2010).

[3] M. S. Arnold, et al., Nature Nanotechnology, 1, 60 (2006).

[4] A. A. Green, et al., Nano Research, 2, 69 (2009).

[5] http://www.nanointegris.com/

[6] M. Engel, et al., ACS Nano, 2, 2445 (2008).

[7] L. Nougaret, et al., Applied Physics Letters, 94, 243505 (2009).

[8] M. Ha, et al., ACS Nano, 4, 4388 (2010).

[9] S. Essig, et al., Nano Letters, 10, 1589 (2010).

[10] A. A. Green and M. C. Hersam, Nano Letters, 8, 1417 (2008).

[11] A. A. Green and M. C. Hersam, Nature Nanotechnology, 4, 64 (2009).

[12] A. A. Green and M. C. Hersam, Nano Letters, 9, 4031 (2009).

[13] A. A. Green and M. C. Hersam, Journal of Physical Chemistry Letters, 1, 544 (2010).

[14] G. M. Mutlu, et al., Nano Letters, 10, 1664 (2010).

[15] A. L. Antaris, et al., ACS Nano, 4, 4725 (2010). 


\title{
SeParation of Single-Wall Carbon Nanotubes by Gel Chromatography
}

\author{
H. Kataura, ${ }^{1,2}$ H. Liu, ${ }^{1,2}$ D. Nishide, ${ }^{1,2}$ S. Fujii, ${ }^{1,2}$ Y. Feng, ${ }^{1,2}$ Y. Urabe, ${ }^{1,2}$ and T. Tanaka ${ }^{1}$ \\ ${ }^{1}$ Nanosystem Research Institute, AIST, Tsukuba 305-8562, Japan \\ ${ }^{2}$ JST, CREST, Japan \\ h-kataura@aist.go.jp
}

For the electronic device applications of single-wall carbon nanotubes (SWCNTs), mixed production of metal and semiconductor phases was one of the most serious problems because they show completely different transport properties. It is known that density gradient ultracentrifugation (DGU) technique can separate metallic and semiconducting SWCNTs with high purity [1]. However, the separation process is so complicated and the scale up is difficult because of the limited rotor capacity. For the industrial application, more effective separation method was desired. Recently, we found a specific interaction between semiconducting SWCNT and agarose, and developed new separation methods using agarose gel [2-4]. These methods realized high purity separation with low cost, high speed, and high efficiency. In the latest separation method, we used only agarose gel beads and two surfactants, sodium dodecyl sulfate (SDS) for metallic SWCNTs and sodium deoxycholate (DOC) for semiconducting SWCNTs. Figure 1 shows the schematic pictures of the separation. The separation procedure is very simple and can be repeated for many times. In this presentation, we will show the recent progress of our separation methods.

[1] M. S. Arnold et al, Nat. Nanotechnol. 1 (2006) 60.

[2] T. Tanaka et al., Appl. Phys. Express 1 (2008) 114001.

[3] T. Tanaka et al., Nano Lett. 9 (2009) 1497.

[4] T. Tanaka et al., Appl. Phys. Express 2 (2009) 125002. 


\title{
LARge-Scale Production of MonOdisperse Carbon Nanomaterials at NanoIntegris
}

\author{
Dr. Nathan Yoder \\ NanoIntegris Inc, 8025 Lamon Ave, Suite 043, Skokie, IL 60077, nyoder@nanointegris.com \\ (www.nanointegris.com)

 \\ Large-scale production of high purity carbon-based \\ nanomaterials has the potential to enable or improve \\ many applications, and to open new directions of \\ academic research [1]. Recently, the Hersam Group \\ developed a scalable and flexible technique for sorting single walled carbon nanotubes by their physical \\ and electronic structure using density gradient ultracentrifugation [2]. NanoIntegris was founded in \\ January 2007 to commercialize this breakthrough technology and make these materials available in large \\ quantities to academic and industrial researchers. During the past 3 years, NanoIntegris has dramatically \\ scaled up production capacity and lowered costs to meet broad and growing global demand for high \\ performance nanomaterials. Today, NanoIntegris supplies materials to over 100 organizations around \\ the world ranging from world-class universities to Fortune 100 companies. Highlights of recent \\ academic journal articles using DGU material will be presented, along with recent progress in the scale- \\ up and commercialization of other nanomaterials at NanoIntegris.
}

[1] M.C. Hersam, Nature Nanotechnology, 3, 387 (2008).

[2] M.S. Arnold, et al., Nature Nanotechnology, 1, 60 (2006).

\section{Understanding THE DNA-BASEd CARbon NANOTUbe CHIRALITy SEPARATion}

\author{
Xiaomin $\mathrm{Tu}$ \\ Polymers Division, National Institute of Standards and Technology, Gaithersburg, MD 20899-0854 \\ xiaomin.tu@nist.gov
}

We have demonstrated in the past that specific DNA can be for the purification of all 12 major semiconducting single-chirality species from a synthetic mixture. We have been investigating the separation mechanism using both experimental and computational approaches. Fluorescence spectroscopy was found to be a sensitive tool to probe the DNA structure on the nanotube, which revealed strong DNA length-dependency of the fluorescence intensity for the recognition sequence motif. Molecular dynamics study suggested DNA wrapping structure involving a novel secondary DNA structure motif. Based on such model, we could predict other sequence motif that may support singlechirality tube enrichment. 


\title{
The Role of Composition-Dependent Catalyst Structure on ChiRAlity Distributions OF As-GROWN SWCNTS
}

\author{
R. Mohan Sankaran \\ Dept. of Chemical Engineering, Case Western Reserve University
}

The exemplary physiochemical properties of single-walled carbon nanotubes (SWCNTs) are largely determined by their diameter and chiral angle, specified as chiral indices (n,m). Despite the significant progress that has been made in purifying mixtures of SWCNTs to obtain monodisperse samples (in terms of chirality), large-scale applications require improvements in the homogeneity of as-grown SWCNTs. Recent reports have shown that a potential route to controlling the chirality distribution of SWCNTs during growth is through catalyst design [1,2]. In this talk, we present our approach which is based on tuning the catalyst composition, independent of size, to perturb the catalyst structure and ultimately influence the SWCNT chirality.

1. Harutyunyan et al., Science 326, 116 (2009).

2. Chiang et al., Nature Mater. 8, 882 (2009).

\section{CARBON NANOTUBES FOR NANOELECTRONICS AND NANOBIOTECHNOLGY}

\author{
Hongjie Dai \\ Department of Chemistry, Stanford University
}

This talk will present our work on using carbon nanotubes for nanoelectronics and biological applications. I will present progress and hurdles in both these areas.

We have combined both controlled nanotube synthesis and chemical separation towards single-chirality SWCNT samples for electronics applications. A major goal in the nanotube electronics area has been obtaining pure semiconducting SWCNTs with a single-chirality, forming parallel arrays of them, and producing devices such as transistor arrays on large substrates.

Carbon nanotubes exhibit many unique intrinsic physical and chemical properties and have been intensively explored for biological and biomedical applications in the past few years. In this talk, I will summarize the main results from our and other groups in this field. Ultrasensitive detection of biological species with carbon nanotubes can be realized. Surface-enhanced Raman spectroscopy of carbon nanotubes opens up a method of protein microarray with detection sensitivity down to $1 \mathrm{fmol} / \mathrm{L}$. In vitro and in vivo toxicity studies reveal that highly water soluble and serum stable nanotubes are biocompatible, nontoxic, and potentially useful for biomedical applications. Carbon nanotube-based drug delivery has shown promise in various in vitro and in vivo experiments including delivery of drugs. Moreover, single-walled carbon nanotubes with various interesting intrinsic optical properties have been used as novel photoluminescence, Raman, and photoacoustic contrast agents for imaging of cells and animals. 


\title{
Performance of Type-separated Single-wall Carbon Nanotubes in ENERGY CONVERSION SCHEMES: CHARGE TRANSFER AND TRANSPORT
}

\author{
Jeff Blackburn, National Renewable Energy Laboratory, Golden, CO \\ Co-authors: Josh Holt, Kevin Mistry, Brian Larsen, John-David Rocha, Andrew Ferguson, Nikos \\ Kopidakis, Drazenka Svedruzic, Paul King, Garry Rumbles
}

Single-wall carbon nanotubes (SWCNTs) have several fundamental properties that make them attractive for sustainable energy conversion technologies, including high electron and hole mobilities, size-tunable ionization potentials and electron affinities in an energy range relevant to many photovoltaic devices, and optical transitions in the visible and near-infrared spectral regions. Additionally, they possess numerous properties amenable to practical, scalable, and economic device fabrication including abundant source material, a natural disposition for solution processing, high surface area for efficient charge transfer, and flexibility. Current projects in our group are aimed at understanding the functionality of SWCNTs in energy conversion technologies where the nanotubes are used to collect and transport charges, and how this functionality varies with SWCNT electronic structure. This presentation will focus on two recent studies. In the first study, we use SWCNTs as interfacial materials to electronically couple a bulk carbon electrode to immobilized hydrogenase enzymes for hydrogen production or oxidation. We demonstrate (1) that SWCNT networks serve as high surface area electroactive scaffolds to which high densities of nano-scale catalysts may be immobilized, and (2) that the SWCNTs perform as molecular wires, efficiently transporting carriers from bulk electrodes to the immobilized catalysts. Furthermore, electrodes prepared with type-enriched SWCNTs show markedly improved hydrogen oxidation/production current densities when enriched with metallic SWCNTs (mSWCNTs). We achieve proton reduction (hydrogen production) and hydrogen oxidation current densities in the range of several $\mathrm{mA} / \mathrm{cm}^{2}$ with $\mathrm{m}$-SWCNT enriched electrodes, a significant improvement over previous hydrogenase-based electrodes.

The second study involves the incorporation of SWCNTs into the active layer of organic photovoltaic (OPV) cells as replacements for the electron accepting fullerene phase. Although replacement of the ubiquitous fullerene acceptors by SWCNTs in OPV devices has shown limited success thus far, the number of fundamental investigations of charge transfer between SWCNTs and conjugated polymers is rather low. It is often assumed (1) that photo-induced charge separation occurs at the interface between SWCNTs and certain conducting polymers, and (2) that m-SWCNTs should limit the generation efficiency and/or lifetime of the charge-separated state. A consideration of the continuous density of states (lack of a true gap) for m-SWCNTs suggests these species should act as recombination centers when interfaced with conducting polymers. To address these points, we use time-resolved microwave conductivity, (TRMC) to probe the generation efficiency and lifetime of charge separation due to the inherent sensitivity of TRMC to free and mobile charge carriers. We first demonstrate photo-induced electron transfer from poly(3-hexylthiophene) (P3HT) to SWCNTs for bulk heterojunctions made with un-enriched SWCNTs. Next, we discuss TRMC results on SWCNT/P3HT bulk heterojunctions containing varying proportions of s- and m-SWCNTs. These studies demonstrate that the proportion of long-lived carriers produced by charge separation can be significantly increased by eliminating the metallic species.

These studies demonstrate the potential utility of SWCNTs in next generation energy conversion schemes and encourage further optimization of these technologies through enrichment of particular electronic structures. 
Fourth NIST Workshop on Carbon Nanotubes: Control and Measurement of Chirality 


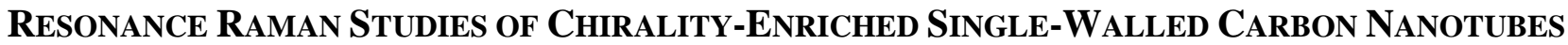

\author{
$\underline{\text { Stephen K. Doorn }}{ }^{1}$, Juan G. Duque ${ }^{1}$, Svetlana Kilina ${ }^{1}$, Sergei Tretiak ${ }^{1}$, Andy Shreve ${ }^{1}$, Hang Chen $^{2}$, \\ Anna Swan ${ }^{2}$, Xiaomin $\mathrm{Tu}^{3}$, Ming Zheng ${ }^{3}$ \\ ${ }^{1}$ Center for Integrated Nanotechnologies, Los Alamos National Laboratory, Los Alamos, NM 87545 \\ ${ }^{2}$ Dept. of Electrical and Computer Engineering, Boston University, Boston, MA 02215 \\ ${ }^{3}$ National Institute of Standards and Technology, Gaithersburg, MD 20899-8540
}

The growing access to nanotube samples that are highly enriched in single chiralities is enabling new photophysical measurements previously impossible at the ensemble level due to spectral congestion from the overlapping responses of different species. In particular, probing chirality-defined electronic behaviors uniquely accessible through resonance Raman spectroscopy of the nanotube G-band requires working at either the single tube level or with ensemble samples highly enriched in a single type of tube structure. We present Raman measurements on such enriched ensemble samples from DNA wrappingbased ion exchange chromatography. Characterization data demonstrating the high level of singlechirality enrichment in these samples will be shown. We also present resonance window behavior of Gband spectra for several single chirality semiconducting species. The Raman excitation profiles at resonance with the $\mathrm{E}_{22}$ transitions allow testing of different models for the Raman scattering process. Strong asymmetries in the profiles reveal new evidence for the importance of non-Condon effects in the Raman response. Results will be discussed in the context of theoretical models that suggest significant coordinate dependence in the transition dipole and phonon-mediated state mixing. Quantum interference between the higher lying $\mathrm{E}_{33}$ and $\mathrm{E}_{44}$ transitions for selected chiralities is also probed using UV resonance Raman at energies up to $3.6 \mathrm{eV}$. The novel behaviors of relative $\mathrm{LO}$ and TO Raman mode intensities and their excitation profiles are discussed in terms of both Raman interference effects and state mixing.

\section{SINGLE-WALLED CARBON NANOTUBES FOR CANCER IMAgING AND THERAPY}

\author{
Xiaoyuan (Shawn) Chen
}

\section{National Institute of Biomedical Imaging and Bioengineering}

Single-walled carbon nanotubes have very unique physicochemical properties suitable for molecular imaging and therapy. This presentation will discuss how to render carbon nanotubes water-soluble and biocompatible, how to load targeting ligands and therapeutic drugs/genes in covalent and non-covalent fashion, and how to image the distribution, fate and therapy efficacy of the composite theranostic nanotube platform. 


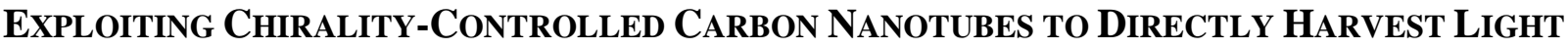 AND Energy Via A Photovoltaic EfFEct on a MACroscopic SCALE
}

\author{
Michael S. Arnold \\ University of Wisconsin-Madison \\ msarnold@wisc.edu
}

Recent breakthroughs in the post-synthetic isolation of chirality-controlled semiconducting carbon nanotubes have made it possible to exploit these exceptional semiconductors as the primary optical absorbing components of macroscopic $10^{11}$ nanotube/ $\mathrm{mm}^{2}$ optoelectronic and photonic devices. Semiconducting carbon nanotubes have extraordinarily attractive properties for harvesting light including unusually strong optical absorptivity at their band gap $\sim 1 \times 10^{6} \mathrm{~cm}^{-1}$ (in thin film); unparalleled charge transport mobility as high as $1 \times 10^{5} \mathrm{~cm}^{2} \mathrm{~V}^{-1} \mathrm{~s}^{-1}$; broad band gap tunability throughout the near- and mid-infrared electromagnetic spectra; self-passivated surfaces that are without dangling bonds or traps; economical solution-processability; and excellent stability to photo-oxidation.

We are specifically interested in exploiting semiconducting carbon nanotubes to create photovoltaic devices such as high-efficiency, economical photovoltaic solar cells and high-detectivity, high-speed photovoltaic photodetectors for optical communications and thermal imaging technologies that are spectrally tunable and integrable onto arbitrary substrates.

The first challenge in creating nanotube-based photovoltaic devices is overcoming the exciton binding energy. We have found that C60-fullerene derivatives and poly(thiophene)s such as poly(3hexylthiophene) induce carbon nanotube exciton dissociation resulting in electron and hole transfer, respectively, away from the nanotubes, for $\mathrm{d}<1.3 \mathrm{~nm}$. The charge separation can be extremely efficient; we have realized combined exciton dissociation, charge transfer, and charge collection efficiency $>80$ \% using C60 as the electron acceptor. Significantly weaker or no charge transfer is observed for optically excited nanotubes paired with poly(para-phenylene vinylene) derivatives, poly(fluorene) derivatives, or insulating polymers such as polycarbonate and polyvinylpyrrolidone due to band/orbital energy offsets that are insufficient to overcome the expected $\cong 0.2 \mathrm{eV}$ nanotube exciton binding energy.

Building on these studies, we have fabricated macroscopic $\left(\mathrm{mm}^{2}\right)$ photovoltaic and photodetector devices with a near-infrared power conversion efficiency of $1.4 \%$ and specific detectivity of $0.4 \times 10^{12}$ Jones at $1.2 \mu \mathrm{m}$, respectively, exclusively using semiconducting nanotubes to harvest the incident electromagnetic radiation. These results are unique from previous uses of carbon nanotubes in macroscopic photovoltaic-type devices, in which the nanotubes have been limited to optically inactive roles such as charge collection or transport. We have also used the devices as tools for characterizing the fundamental diameter-dependent physical driving forces for charge separation at the nanotube/semiconductor heterointerfaces and the migration of excitons in percolating nanotube networks via intra-tube diffusion and inter-tube hopping. We show that the later limits the overall device efficiency and can be significantly enhanced through chirality control. 


\title{
GROWTH AND SEPARATION OF CHIRALITY CONTROLLED SWCNTS
}

\author{
Sivaram Arepalli \\ Department of Energy Science, Sungkyunkwan University, Suwon, Korea
}

Many of the practical applications of single wall carbon nanotubes (SWCNTs) require either semiconducting or metallic tubes. Purely metallic carbon nanotubes can help develop flexible and portable energy production and storage devices. This presentation will focus on producing and separating the single wall carbon nanotubes by "tube type" and chirality [1,2]. Increased arm chair tube populations are observed when a "warm up" laser is used to prolong the nucleation process in a modified double pulse laser oven set up. Similar controlled growth was achieved by using different metal catalysts $(\mathrm{Pt} / \mathrm{Rh}$ instead of $\mathrm{Co} / \mathrm{Ni})$. The talk will also discuss some of the results from our recent “type separation” experiments carried out using agarose gel and ultracentrifugation.

\section{References:}

P. Nikolaev, W. Holmes, E. Sosa, S. Arepalli and L. Yowell, "Effect of Vaporization Temperature on the Diameter and Chiral Angle Distributions of Single Wall Carbon Nanotubes”, J. Nanoscience and Nanotechnology, Vol. 10, 3780-3789 (2010).

P. Nikolaev, W. Holmes, E. Sosa, P. Boul, and S. Arepalli, "Effect of the Laser Heating of Nanotube Nuclei on the Nanotube Type Population”, Nano Research Vol. 2, pp. 818-827 (2009). 
Poster Abstracts

Thursday, September $23^{\text {rd }}, 2010$ at 5 pm - Hall of Flags

\title{
INVESTIGATE THE DNA RECOGNITION SEQUENCES FOR CARBON NANOTUBE SEPARATION BY FLUORESCENCE SPECTROSCOPY
}

\author{
Xiaomin Tu, Ming Zheng \\ Polymers Division, National Institute of Standards and Technology, Gaithersburg, MD 20899-0854 \\ xiaomin.tu@nist.gov
}

Fluorescence spectroscopy is a powerful characterization tool for semiconducting carbon nanotube. It can distinguish nanotubes of similar diameter with overlapping $E_{11}$ energy transitions, and it is very sensitive to surrounding changes, e.g. dielectric environment. We have identified a set of DNA sequences that can enrich single-chirality carbon nanotubes through a systematic search, and we adopted fluorescence spectroscopy to analyze these recognition sequences dispersed carbon nanotubes for mechanistic understanding of DNA-based nanotube separation. The results revealed strong DNA-length dependency for the recognition sequences. We believe fluorescence spectroscopy can be a very useful technique to unveil the separation mechanism.

\section{Elemental ANalysis of a Single-Walled Carbon Nanotube Candidate RefERenCE Material Using Instrumental and Cold NeUtron Prompt GaMma ACtivation ANaLYSIS}

\author{
Rabia Oflaz Spatz, Rolf Zeisler, and Rick Paul \\ National Institute of Standards and Technology, Chemical Science and Technology Laboratory, \\ Gaithersburg, MD 20899
}

A material containing single-wall carbon nanotubes (SWCNTs) mixed among other carbon species, catalyst residues, and trace element contaminants has been prepared by the National Institute of Standards and Technology for characterization and distribution as Standard Reference Material (SRM 2483 Carbon Nanotube Soot). Neutron metrology was used in the characterization of elemental content of the candidate SWCNT reference material. The neutron techniques include instrumental neutron activation analysis (INAA) and cold neutron prompt gamma activation analysis (CNPGAA). Results will be discussed in the presentation. 


\title{
Electrical Reliability Testing OF Single-W Alled CaRbon NANOtUbe Networks
}

$\underline{\text { Mark C. Strus }}^{\dagger}$, Ann Chiaramonti-Debay ${ }^{\dagger}$, Younglae Kim ${ }^{\S}$, Yung Joon Jung , Robert R. Keller ${ }^{\dagger}$

†National Institute of Standards and Technology, Materials Reliability Division, Boulder, CO 80305

$\S$ Electrical and Computer Engineering, Northeastern University Boston, MA 02115

- Mechanical and Industrial Engineering, Northeastern University Boston, MA 02115

mark.strus@nist.gov

Because of their high current carrying capability and appreciable thermal conductivity, single-walled carbon nanotubes (SWCNTs) have emerged as a ideal material for future nanoscale electronics. The unique quasi-one-dimensional shape and strong sp2 and $\pi$-bonding between neighboring carbon atoms allows for large electron mean free paths, which means CNTs can conduct ballistically while withstanding extreme current densities $>10^{9} \mathrm{~A} / \mathrm{cm}^{2}$. SWCNT networked films, which can be easily fabricated at low cost, are emerging as a new class of flexible electronics with applications as field effect transistors, chemical sensors, and transparent oxide layers in photovoltaics. However, the long-term performance and reliability of these CNT-based devices has been largely neglected despite the likely subjection to high temperatures from significant currents which may directly induce thermal failure, cause electromigration, or lead to thermal mechanical induced fatigue or separation at the critical CNT$\mathrm{CNT} / \mathrm{metal} /$ dielectric/polymer interfaces.

We present test methods that are used to investigate the electrical reliability of several similar nanoscale lines of highly aligned networked metallic and semiconducting SWCNTs fabricated through a templatebased fluidic assembly process. We find that these SWCNT networks can withstand current densities on the order of $1 \mathrm{MA} / \mathrm{cm}^{2}$ for several hours and in some cases, several days, though the rate of failure and total lifetime is dependent on the fabrication conditions as demonstrated by scanning electron microscope (SEM) and transmission electron microscope (TEM) cross-sectional images. Well-fabricated lines subjected to constant electrical stress are found to fail at a linear rate until moments before opencircuit failure, and we offer suggestions on the possible types of failure mechanisms involved.

\section{SEPARATION AND CHARACTERIZATION OF LARGE DIAMETER EMPTY AND WATER-FILLED SWCNTS}

\author{
Jeffrey A. Fagan, ${ }^{1}$ Ji Yeon Huh, ${ }^{1}$ Jeffrey R. Simpson, ${ }^{2}$ Jeffrey Blackburn, ${ }^{3}$ \\ Angela R. Hight Walker ${ }^{4}$ \\ 1: National Institute of Standards and Technology: Polymers Division \\ 2: Towson University, Towson MD \\ 3: National Renewable Energy Laboratory, Golden CO \\ 4: National Institute of Standards and Technology: Optical Technology Division
}

We report measurements of separated populations of water-filled and empty nanotubes in solution and a facile method for separating the distinct populations from the initial mixture. In particular, results are shown for separation of laser ablation and electric arc synthesis produced nanotubes with diameters ranging from $1.2 \mathrm{~nm}$ to $1.5 \mathrm{~nm}$ using deoxycholate (DOC) surfactant based on the absence or presence of liquid in the interior cavity. Isolation of these populations was achieved from multiple SWCNT populations obtained from several independent synthesis sources. The separated populations are then demonstrated to have different optical resonance energies, radial breathing mode locations, and fluorescence efficiencies, as well as different optimal conditions for metallic and semiconducting separation. 


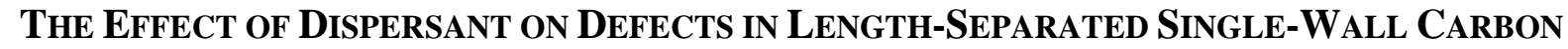 NANOTUBES MEASURED BY RAMAN SPECTROSCOPY

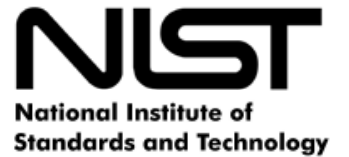 \\ Jeff R. Simpson ${ }^{\mathrm{a}, \mathrm{b}}$, Jeff A. Fagan ${ }^{\mathrm{c}}$, Matthew L. Becker ${ }^{\mathrm{d}}$, Erik K. Hobbie ${ }^{\mathrm{e}}$, Angela R. Hight Walker ${ }^{\mathrm{a}}$

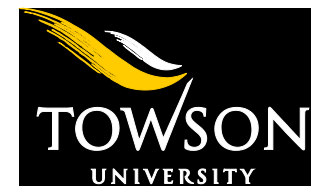

${ }^{a}$ Physics Laboratory, National Institute of Standards \& Technology (NIST), Gaithersburg, MD 20899,USA

${ }^{b}$ Department of Physics, Astronomy, and Geosciences, Towson University, Towson, MD 21252, USA

${ }^{c}$ Materials Science and Engineering Laboratory, NIST, Gaithersburg, MD 20899, USA

${ }^{d}$ Dept. of Polymer Science, University of Akron, Akron, Ohio

${ }^{e}$ Dept.of Physics, Dept. of Coatings and Polymeric Materials, North Dakota State Univ., Fargo, ND

To evaluate the role of defects in this dependence, we compare Raman spectra from aqueous suspensions of SWCNTs dispersed using either polymer adsorption of single-stranded DNA or micelle encapsulation with sodium deoxycholate surfactant [1]. Each dispersed sample is then separated by length, using either size exclusion chromatography or density-gradient ultracentrifugation for DNA- and DOC-dispersed SWCNTS, respectively. Separation by length affords examination of the relative contribution of intrinsic and internal defects to the Raman D-band. For each dispersion method we examine length-separated fractions ranging from approximately (50 to 1000) $\mathrm{nm}$. The intensity ratio of the disorder-induced Raman D-band to the G'-band scales inversely with length, consistent with predominately end cap defect sites. While the fluorescence quantum yield of SWCNTs in solution depends dramatically on dispersant, the comparable length-dependent $D / G$ ' for each dispersion scheme excludes defects as a possible explanation for the lower fluorescence in DNA versus deoxycholate dispersions.

[1] J.R. Simpson, J.A. Fagan, M.L. Becker, E.K. Hobbie, and A.R. Hight Walker, Carbon 47, 3238 (2009).

\section{Thermogravimetric Analysis of NIST's Single-Wall Carbon Nanotube REFERENCE MATERIAL}

\author{
Elisabeth Mansfield, Stephanie Hooker \\ NIST, Materials Reliability Division, Boulder CO
}

Single-walled carbon nanotubes (SWCNTs) are a promising nanomaterial with applications to aerospace, electronics and biotechnology. However, the properties of different batches of carbon nanotubes can vary considerably depending on chemical purity and the nanotube types present (e.g. diameter and chirality distribution). Thermogravimetric analysis (TGA) provides one measure of nanotube purity by assessing the material's thermal stability (i.e. how it oxidizes with temperature) by monitoring weight loss as a function of temperature. Evaluation of the NIST Standard Reference Material for SWCNTs has been evaluated with TGA. Results of the raw soot, evaluated for composition with TGA will be presented. Length-sorted fractions of the SWCNT reference material (long, medium, short) will also be available and TGA characterization of each of these fractions will be demonstrated. 


\title{
Electrical Conductivity Measurements of Individual Single-Wall CaRbon NanOtubeS AND Transparent Conducting CARbon NANOTUbE FiLMS
}

\author{
Jan Obrzut \\ NIST, Polymers Division, Gaithersburg, MD 20899-8541
}

Carbon nanotubes are being considered for electronic devices that can be scaled down to nanodimensions. The electrical transport properties of carbon nanotubes are especially attractive since they are not affected by surface scattering or surface roughness when the feature size of the interconnects shrinks. We describe impedance measurements of individual single wall metallic carbon nanotubes (CNTs) in the frequency range of $40 \mathrm{~Hz}$ to $100 \mathrm{MHz}$. Model calculations for carbon nanotubes predict that a drop in impedance should occur due to damping of the plasmon wave at a crossover frequency $f_{c}=$ $1 / \pi R_{D C} C_{Q E}$ where $R_{D C}$ is the resistance and $C_{Q E}$ are the combined quantum and electrostatic capacitance of the tube. In our earlier work we showed that the broad-band impedance measurement can be used to separate resistance from the capacitance of individual CNTs assembled as a conducting channel of a 3terminatl field-effect-transistor (FET) structure. Our present work is aimed at further elucidating the effect of contact barriers on the crossover frequency and the measurement of capacitance of CNTs. We utilized a resistance - capacitance (RC) lumped element circuit model to extract the capacitance of the tube and the corresponding contact resistance. The resistance values are approximately symmetric in respect to \pm DC bias indicating symmetric contacts that can be attributed to forward-biased Schottky barriers at each contact. We observe a sharp conductor-insulator transition at a crossover frequency, $f_{c}$, above which the circuit response becomes capacitive. Our results agree qualitatively with the theoretical impedance characteristic of a perfect metallic CNT, and furthermore, they imply that the crossover frequency due to quantum capacitance would fall in the range of about $100 \mathrm{GHz}$.

Transparent conducting films were made from length-sorted single wall carbon nanotubes through filtration from a dispersing solvent onto a filter substrate. Such films exhibit sharp changes in their optical properties and conductivity $(\sigma)$ with increasing surface concentration of the tubes. At a given surface concentration, tubes longer than $200 \mathrm{~nm}$ are found to form networks that are more transparent and conducting. We show that changes of $\sigma$ with tube concentration can be quantitatively described by the Generalized Effective Medium theory. The scaling universal exponents describing the 'percolation' transition from an insulating to conducting state with increasing concentration are consistent with twodimensional (2D) percolation model. Shorter tubes and mixed length tubes form 3D networks.

Furthermore, we demonstrate that the conductivity percolation threshold $\left(x_{c}\right)$ varies with the aspect ratio $L$ as, $\mathrm{x}_{\mathrm{C}} \sim 1 / L$, a result that is also in accordance with the percolation theory. These findings provide a framework for engineering the optical and electrical properties of carbon nanotube networks for technological applications where flexibility, transparency and conductivity are required. 


\title{
Controllable Synthesis of Single-WALled CARbon NANOtubES AND GRAPHENE IN ARC DischARGE
}

Jian Li, Olga Volotskova, Alexey Shashurin, Madhusudhan Kundrapu and Michael Keidar Department of Mechanical and Aerospace Engineering, The George Washington University, Washington, DC 20052, USA

Arc charge is the most widely-utilized method to synthesize carbon nanotubes and other carbon nanostructures due to the high-quality products and eco-friendly processes. The application of magnetic field in arc can increase the controllability and flexibility of the synthesis processes. The focus of this work is to understand the mechanism of magnetically-enhanced plasma synthesis, further to establish the fundamental correlation between parameters of arc plasma and characteristics of single-walled carbon nanotubes (SWCNT) and graphene. The influence of external magnetic field on SWCNT parameters is demonstrated as following: (I) It can increase the length of SWCNT by a factor of 2 and the population of long nanotubes with the length above $5 \mu \mathrm{m}$. (II) It can result in substantial fractions of produced SWCNTs being of small diameter, less than $1.3 \mathrm{~nm}$. (III) It can change the chirality distribution of SWCNT and the ratio of metallic to semiconducting SWCNT. (IV) It can reduce the diameter of catalyst nanoparticles and narrow the diameter distribution of nanoparticles. The explanations of these findings can be presented in the study of voltage-current characteristics of arc plasma, the analysis of size distribution of catalyst particles, the diffusion model of carbon adatom by Monte Carlo and numerical simulation of arc discharge ablation. Additionally, recent research results in our group show that by applying non-uniform magnetic field, large-scale production of high-quality graphene flakes can be synthesized on Molybdenum sheet. The morphology and quality of the carbon nanostructures are characterized by scanning electron microscopy (SEM), TEM, Raman, electron diffraction spectrum, UV-visible near-infrared absorbance and near-infrared fluorescence spectroscopy.

\section{SELF-ASSEMbly OF CARBon NANOTUBES FOR LENGTH FraCtionAtion AND ChARACTERIZATION}

\author{
Constantine Y. Khripin ${ }^{1}$, Nicholas Arnold-Medabalimi ${ }^{2}$, Denis Pristinski ${ }^{1}$, and Ming Zheng $^{1}$ \\ ${ }^{1}$ Complex Fluids Group, Polymers Division, National Institute of Standards in Technology, 100 Bureau Dr, \\ Gaithersburg, MD 20899; 'Summer High School Internship Program', National Institute of Standards in \\ Technology, 100 Bureau Dr, Gaithersburg, MD
}

The DNA dispersion of single - walled carbon nanotubes, forming the hybrid nanoparticle DNASWCNT, has seen extensive use as a convenient way of purifying and processing carbon nanotubes. We are investigating molecular self-assembly as a new way of purifying DNA-SWCNT, as well as for probing the structure of the hybrid. We find that the addition of poly-ethyleneglycol (PEG) induces DNA-SWCNT assembly into a gel - like phase. This is similar to the PEG "molecular crowding” induced transition of double-stranded DNA into a condensed phase. As with DNA, DNA-SWCNT condensation is length dependent; AFM measurements show both long and short nanotubes can be isolated, e.g. $460 \pm 180 \mathrm{~nm}$ and $160 \pm 50 \mathrm{~nm}$. Dynamic light scattering and UV-VIS data show that this method removes non-SWCNT impurities present in the suspension. We also show that "molecular crowding" by PEG can induce DNA-SWCNT self-assembly into a 2D nematic phase on a substrate. Because this phase appears to be very stable, we are exploring its use for nanotube characterization by scanning probe microscopy, to examine the structure of DNA on the nanotube surface. 


\title{
Wrinkling AND Strain Softening in Single-Wall CaRbon Nanotube MeMbraneS on Polymer Substrates
}

\author{
Erik K. Hobbie \\ Department of Physics, Department of Coatings and Polymeric Materials, North Dakota State University \\ Daneesh O. Simien, Jeffrey A. Fagan, Ji Yeon Huh, Jun Young Chung, Steven D. Hudson, Jan Obrzut, \\ Jack F. Douglas and Christopher M. Stafford \\ Polymers Division, NIST Gaithersburg
}

Thin membranes of single-wall carbon nanotubes (SWCNTs) show considerable promise for a number of potential applications. The high conductivity and shape anisotropy of the nanotubes enable the formation of conductive quasi-2D networks at remarkably low surface density, and the mechanical characteristics of the individual SWCNTs can be outstanding. Recent advances in the separation of SWCNTs by length and electronic type allow for the production of films and coatings with precisely tunable properties, and the tremendous potential of these films for flexible-electronics applications demands a deeper understanding of the coupling between deformation, microstructure and stiffness. Compressive wrinkling has emerged as a powerful tool for measuring the modulus of thin polymer films supported by soft flexible substrates, and we use this approach here to study the nonlinear deformation of membranes assembled from SWCNTs that have been purified by length or electronic type (metallic vs. semiconducting). Our measurements reveal a material that is remarkably stiff under infinitesimal deformation but softens dramatically at finite strain. We link this strongly non-linear behavior to an upward shift in percolation threshold triggered by strain-induced nanotube alignment, an effect correspondingly apparent as an anisotropic decrease in conductivity. We extract the modulus and yield strain as a function of surface density and film thickness and compare these with theoretical models of percolation in anisotropic 2D rigid-rod networks.

This work is supported by the National Science Foundation through CMMI-0969155.

\section{DOCUMENTARY STANDARDS DEVELOPMENT FOR CARBon NANOTUBE CHARACTERIZATION WITHIN ISO/TC 229}

\author{
Angela R. Hight Walker
}

Chair of US Delegation to ISO TC229/WG2, National Institute of Standards and Technology

Carbon nanotubes (CNTs) play a prominent role within the International Standards Organization (ISO), Technical Committee (TC) 229 Nanotechnology. ISO/TC229, established in 2005, has 32 member body countries participating and 10 countries observing, and works in cooperation with multiple organizations such as CEN, OECD, VAMAS, Asia Nano Forum, BIPM, IUPAC among others. CNTs are the focus of the vast majority of work items within Working Group 2 (WG2), Measurement and Characterization, one of the four WGs within the TC. WG2 is a joint committee with the International Electrotechnical Commission (IEC)/TC 113. The work program for single-wall carbon nanotubes is based on a matrix of six parameters verses multiple measurement methods. Many of these technique-based projects have moved into the final stages of development. Also, multiwall carbon nanotube characterization is under consideration within WG2. A summary of the documentary standards under development pertaining to carbon nanotubes will be presented, particularly those within ISO/TC229, but also those relevant activities in other standards developing organizations. 


\title{
ENRICHMENT OF ARMCHAIR CARBON NANOTUBES VIA DENSITY GRADIENT UlTRACENTRIFUGATION: Full Population Analysis using Raman Excitation Profiles
}

Erik H. Hároz ${ }^{1}$, William D. Rice ${ }^{1}$, Benjamin Y. Lu ${ }^{1}$, Georgia K. Lagoudas ${ }^{1}$, Robert H. Hauge ${ }^{2}$, Stephen K. Doorn ${ }^{3}$, and Junichiro Kono ${ }^{1}$

${ }^{1}$ Department of Electrical and Computer Engineering, Rice University

${ }^{2}$ Department of Chemistry, Rice University

${ }^{3}$ Center for Integrated Nanotechnology, Los Alamos National Laboratory

Using resonant Raman scattering spectra collected over a broad range of excitation wavelengths (440$850 \mathrm{~nm}$ ), we have constructed Raman excitation profiles of the radial breathing mode phonon for each $(n, m)$ species present in as-produced and metal-enriched single-walled carbon nanotube ensemble samples. From this, we determine the relative abundances of all metallic and semiconducting chiralities. Strikingly, the data clearly show that our density gradient ultracentrifugation (DGU) process enriches the metal-enriched sample in armchair and near-armchair species. In particular, we observe that armchair carbon nanotubes constitute more than $50 \%$ of each metallic $(2 n+m)$ family yet observe little to no chiral angle dependence in the remaining semiconducting species minority. Such data combined with absorption and photoluminescence measurements elucidate elements of the mechanism of the DGU metallic type-enrichment process and the importance of surfactant micelle composition. Finally, we compare our measured relative abundances determined from Raman spectroscopy to absorption area estimates to assess the validity of the usage of absorption spectroscopy for determining \% metallicity of ensemble nanotube samples.

\section{Resonant Raman Scattering Behavior of G-Band Phonons in ARMchair-EnRiched Carbon NANOTUBE SUSPENSIONS}

\author{
Erik H. Hároz ${ }^{1}$, Juan G. Duque ${ }^{2}$, William D. Rice ${ }^{1}$, Stephen K. Doorn ${ }^{2}$, and Junichiro Kono ${ }^{1}$ \\ ${ }^{1}$ Department of Electrical \& Computer Engineering, Rice University, Houston, TX \\ ${ }^{2}$ Center for Integrated Nanotechnologies, Los Alamos National Laboratory, Los Alamos, NM
}

The emission of longitudinal and transverse optical phonons (LO \& TO, respectively), which make up the so-called "G-band" of carbon nanotubes (CNTs), are thought to be important to electrical and optical relaxation processes in such systems because of their strong electron-phonon coupling. The standard tool for probing this coupling, especially in metallic carbon nanotubes, is resonant Raman scattering spectroscopy. Unfortunately, unlike the radial breathing mode (RBM) in CNTs, however, the G-band does not display a strong frequency dependence on $(n, m)$ species and hence nanotube structure. As a result, obtaining a clear chirality dependence in terms of peak frequency, peak intensity, and line width of the $\mathrm{LO}$ and TO phonons of the G-band requires working at either the single tube level or with ensemble samples highly enriched in a single type of tube structure. Here, we present Raman measurements on ensemble samples enriched in metallic carbon nanotubes produced by density gradient ultracentrifugation (DGU) and provide clear evidence that our DGU process enriches in particular armchair metallic chiralities. Furthermore, G-band data for spectroscopically isolated armchair chiralities show that the relevant lower-frequency LO mode is absent for these structures, in contrast with recent theoretical results. 


\title{
Phonon Dephasing and Population Decay Dynamics of the G-Band of Semiconducting Single-WALl CARbon NANOTUBES
}

Young Jong Lee, Sapun H. Parekh, Marcus T. Cicerone, and Jeffrey A. Fagan

Polymers Division, National Institute of Standards and Technology, Gaithersburg, MD 20899

The dephasing and population decay dynamics of optical phonons are studied for semiconducting single-wall carbon nanotubes (SWCNTs) using broadband time-resolved coherent anti-Stokes Raman scattering (TR-CARS) and time-resolved incoherent anti-Stokes Raman scattering (TR-IARS). By simply adjusting the spectral bandwidth of a continuum pulse, we are able to directly measure both the total dephasing time, $T_{2}$, and the population decay time, $T_{1}$, of the G-band sequentially in the same sample, which allows for one to exclude artifacts due to comparison of dynamics values measured with different sample conditions and different measurement schemes. The values of $T_{1}$ and $T_{2} / 2$ are presented for two different SWCNT samples: bundles in a film on glass and a dispersion solution in water. While the measured $T_{1}$ values are similar for the two samples, the pure dephasing times, $T_{2}{ }^{*} / 2$, determined from the $T_{2} / 2$ and $T_{1}$ measurements are faster in bundled SWCNTs than in an isolated dispersion. This suggests that neighboring tubes in the film perturbs the vibrational mode more strongly than surrounding surfactants and that the pure dephasing dynamics is more sensitive to the perturbation.

\section{CARbon NANOtUbE REFERENCE MATERIALS}

\author{
Jeffrey A. Fagan \\ National Institute of Standards and Technology, Polymers Division, Gaithersburg, MD 20899 \\ jeffrey.fagan@nist.gov
}

NIST has produced and characterized two different carbon nanotube reference materials for upcoming release. These include a certified reference material based on a homogenized batch of raw nanotube soot (SRM 2483) and a set of purified length sorted populations in aqueous dispersion (RM 8281). SRM 2483 will be certified for the elemental composition and homogeneity of the material as evaluated by neutron activation analysis, inductively coupled plasma - mass spectometry (ICP-MS), and thermogravimetric analysis; informational values with respect to the optical absorbance of the material after dispersion, Raman scattering information, and TEM images will also be detailed. RM 8281, also in use as the round robin sample for the VAMAS TWA 34 project \#1 on chirality distribution measurement, will have information including optical properties, and the distribution of lengths within the three fractions. An overview of the measured properties is presented. 


\title{
Single Wall Carbon Nanotube Processing Technologies and ApPlications
}

\author{
Christopher M. Schauerman, Paul R. Jarosz, Matthew J. Ganter, Roberta A. DiLeo, Thomas L. \\ Mastrangelo, Jack Alvarenga, Cory D. Cress ${ }^{\dagger}$, Ryne P. Raffaelle ${ }^{\ddagger}$, \\ and Brian J. Landi \\ NanoPower Research Laboratories, Rochester Institute of Technology, Rochester, NY, USA \\ ${ }^{\dagger}$ Naval Research Laboratories, Washington, DC, USA \\ ${ }^{\ddagger}$ National Center for Photovoltaics, National Renewable Energy Laboratories Golden, CO, USA
}

Many applications of single wall carbon nanotubes (SWCNTs) stand to benefit from advanced techniques for isolating carbon nanotubes on the basis of electronic type, diameter, and length. In response to the growing demand for material, recent efforts have focused on improving the yield and efficiency of these separation tools. One strategy for increasing the mass efficiency of the separation process is to influence the SWCNTs distributions (e.g. chirality, diameter, length, etc.) upstream by modifying synthesis, purification, and processing parameters. With precise optimization of these procedures, a larger mass fraction of nanotubes can be retained throughout the processing lifecycle. However, there are currently many challenges regarding SWCNT quality control, which range from reproducibility in synthesis to eventual integration into device structures. This presentation will highlight the versatility of using laser vaporization synthesis to improve SWCNT yields and reproducibility. The laser vaporization method allows for highly consistent chirality and diameter distributions, as well as the ability to shift those distributions during synthesis by controlling reactor parameters such as catalyst selection, carrier gas, temperature, etc. The results of a rapid purity assessment protocol for SWCNT materials (produced by a variety of methods including laser vaporization) based upon dispersion in organic solvents will be described. Utilizing this protocol, an advanced thermal oxidation profiling technique was developed to maximize nanotube mass retention during purification. It is possible to leverage these and other recent advances in SWCNT synthesis and processing techniques to tailor the bulk properties for specific technologies and applications. A summary of demonstrations of high purity SWCNTs in devices will be provided, including thin-film transistors, bulk CNT wires, and lithium-ion batteries. The influence of purity, chirality, and SWCNT morphology are shown to dramatically influence the relative improvement gained in these device architectures. In addition, the effects of metal-nanotube junctions and chemical dopants to enhance the intrinsic SWCNT properties will be discussed. 


\title{
Solid-State ${ }^{13}$ C NMR AssignMent of CARbon ResonanCES ON METAllic AND SEMICONDUCTING Single-WALlED CARBON NANOTUBES
}

\author{
Chaiwat Engtrakul, Brian Larsen, Kevin Mistry, Mark Davis, Jeff Blackburn \\ National Renewable Energy Laboratory, Golden, CO
}

Exploitation of the unique electronic properties of single-walled carbon nanotubes (SWCNTs) in device fabrication requires molecular-level structural characterization of the nanotubes and their derivatives. Among the various analytical tools that can be used to characterize the structural properties of SWCNTs, nuclear magnetic resonance (NMR) spectroscopy is particularly well suited to probe the local electronic environment of carbon nuclei. A major challenge in obtaining a high-resolution ${ }^{13} \mathrm{C}$ NMR spectrum for pristine SWCNTs is the large polydispersity in diameter, length, and chirality intrinsic to most samples. In addition, as-produced SWCNTs consist of $\cong 2 / 3$ semiconducting (s) and $\cong 1 / 3$ metallic (m) nanotubes, and definitive assignment of distinct ${ }^{13} \mathrm{C}$ resonances for each of these types of tubes has not been achieved experimentally.

We report here the first solid-state ${ }^{13} \mathrm{C}$ NMR chemical shift assignment of nanotube carbons on $\mathrm{m}$ - and s-SWCNTs for samples with widely varying s-SWCNT content and diameters of $\sim 1.3 \mathrm{~nm}$, including samples enriched with nearly $100 \% \mathrm{~m}$ - or s-SWCNTs. ${ }^{8}$ We prepare ${ }^{13} \mathrm{C}$-enriched SWCNTs by laser vaporization using graphite targets doped with $20 \%$ amorphous ${ }^{13} \mathrm{C}$. The resulting ${ }^{13} \mathrm{C}$-enriched SWCNTs are then separated by density gradient ultracentrifuation. Solid-state magic angle spinning (MAS) NMR is then used to probe the unique nuclear environment of carbons in the separated SWCNTs. There are two striking features to the MAS NMR spectra: (1) the difference between the ${ }^{13} \mathrm{C}$ NMR resonances between the nearly pure m- and s-SWCNTs is very small, $\cong 1 \mathrm{ppm}$, and (2) the full width at half maximum (FWHM) of the metallic ${ }^{13} \mathrm{C}$ resonance is nearly twice that of the semiconducting ${ }^{13} \mathrm{C}$ resonance. The first observation is in stark contrast to both theoretical and experimental studies that predict and infer, respectively, ${ }^{13} \mathrm{C}$ NMR chemical shifts of up to $11 \mathrm{ppm}$ between m- and s-SWCNTs. The small chemical shift difference observed in our work is in good agreement with the recent theoretical calculations by Lai et al. Here, larger diameter semiconducting and semimetallic zigzag SWCNTs were calculated to have similar isotropic chemical shifts, (121.8 and 120.9) ppm, respectively. We tentatively attribute the increased line broadening of the metallic ${ }^{13} \mathrm{C}$ resonance to polydispersity in the isotropic Knight shift of the different m-SWCNT species. Nonetheless, the FWHMs for the m- and s-SWCNT samples are among the lowest reported values to date.

Our results indicate that high-resolution ${ }^{13} \mathrm{C}$ NMR can resolve m- and s-SWCNTs in mixed SWCNT samples with diameters of $\cong 1.3 \mathrm{~nm}$ if the samples are very pure (i.e. low in residual catalyst metal content). We are currently in the process of exploring smaller diameter SWCNTs to see if both electronic structure and diameter affect the chemical shift. Beyond the fundamental importance of unique diameter- and electronic structure-dependent ${ }^{13} \mathrm{C}$ chemical shifts, the ${ }^{13} \mathrm{C}$-enriched SWCNTs produced here are useful for probing molecular-scale interactions that are important for emerging applications. Such interactions include ground state charge transfer interactions and functionalization reactions that influence separations, as well as interactions with species such as quantum dots and conducting polymers. 


\title{
Characterization of PARTicle Suspensions USing ANAlytical Ultracentrifugation
}

\author{
Vinayak Rastogi and Jeffery A. Fagan
}

National Institute of Standards and Technology, 100 Bureau Dr, Gaithersburg MD 20899

Analysis of disperse systems has been of primary importance for the development of technology based on nano/micro particle suspensions. Several techniques like field flow fractionation, spectroscopy, size measurements based on zeta potential have been used to determine the properties of colloid particle systems. However each presents its own limitations and complexity based on the system of interest. Analytical Ultracentrifuge on the other hand allows dynamic scanning, analysis and characterization of wide variety of particle suspensions under the influence of controllable sedimentation forces. ${ }^{1}$ The technique facilitates the investigation of physico-chemical and hydrodynamic properties of nanoparticle and dispersant conjugates. This poster highlights the utility of analytical ultracentrifugation in the characterization of biomolecules ${ }^{2}$ (DNA, BSA), polymeric microspheres (Latex), metallic nanoparticles ${ }^{3}$ (Si-NP and Ag-NP) and SWCNT-surfactant complexes ${ }^{4}$.

\section{References:}

1. Cölfen, H. Analytical Ultracentrifugation. Macromol. Biosci. 10, 687-688 (2010).

2. Lebowitz, J., Lewis, M.S. \& Schuck, P. Modern analytical ultracentrifugation in protein science: A tutorial review. Protein Sci. 11, 2067-2079 (2002).

3. Jamison, J.A. et al. Size-Dependent Sedimentation Properties of Nanocrystals. ACS Nano 2, 311-319 (2008).

4. Arnold, M.S., Suntivich, J., Stupp, S.I. \& Hersam, M.C. Hydrodynamic Characterization of Surfactant Encapsulated Carbon Nanotubes Using an Analytical Ultracentrifuge. ACS Nano 2, 2291-2300 (2008).

\section{Separation and Characterization of Double-Wall Carbon Nanotube Subpopulations}

\author{
Ji Yeon Huh ${ }^{1}$, Angela R. Hight Walker ${ }^{2}$, Hyun Wook Ro ${ }^{1}$, Jan Obrzut ${ }^{1}$, Elisabeth Mansfield ${ }^{3}$, Roy \\ Geiss $^{3}$, and Jeffrey A. Fagan ${ }^{1}$ \\ National Institute of Standards and Technology, Gaithersburg, Maryland 20899 \\ ${ }^{1}$ Polymers Division, \\ ${ }^{2}$ Physics Laboratory \\ National Institute of Standards and Technology, Boulder, Colorado 80305 \\ ${ }^{3}$ Materials Reliability Division
}

Surfactant-encapsulated double-wall carbon nanotubes (DWCNTs) synthesized by the high-pressure carbon monoxide decomposition (HiPco) process were separated by length and electronic characteristics. To ensure our study focuses only on the behavior of DWCNTs, dispersed DWCNTs were first purified from polydisperse mixtures of SWCNTs, MWCNTs, and carbonaceous impurities following the method of Green et al. [Green, A. A.; Hersam, M. C. Nat. Nanotechnol. 2008, 264, 1]. By increasing the density difference between the nanotubes and the density gradient medium, we exploited the length-dependent translation of the nanotubes in response to applied centrifugation to isolate narrow length distribution The controlled addition of cosurfactants is shown to allow resolution of DWCNTs by electronic structure, as demonstrated through optical absorbance, Raman spectra, and electrical conductivity measurements. Measurements of conducting films prepared from separated fractions exhibit significant property differences in the enriched materials. 


\title{
Uptake ANd Elimination Behaviors of Polyethyleneimine (Pei)-CoAted Multi-Walled CARBon NANOTUBES BY EISENIA FoETIDA AND DAPHNIA MAgNA
}

\author{
Elijah J. Petersen ${ }^{1}$, Roger A. Pinto ${ }^{2}$, Danielle J. Mai ${ }^{2}$, Peter F. Landrum ${ }^{3}$, Liwen Zhang ${ }^{4}$, Qingguo \\ Huang ${ }^{4}$, Xiangyang $\mathrm{Shi}^{5}$, and Walter J. Weber, $\mathrm{Jr}^{2}$ \\ 1:Biochemical Sciences Division, NIST, Gaithersburg, MD, 20899, USA \\ 2:Department of Chemical Engineering, University of Michigan, Ann Arbor, MI, 48109, USA \\ 3: National Oceanic and Atmospheric Administration, Great Lakes Environmental Research Laboratory, 4840 \\ State Rd., Ann Arbor, MI, 48108, USA \\ 4:Department of Soil Sciences, University of Georgia, Griffin, GA, 30223 \\ 5: Nanobiotechnology Laboratory, College of Chemistry, Chemical Engineering, and Biotechnology, Donghua \\ University, Shanghai 201620, P. R. China
}

Determining the effects of various surface coatings on carbon nanotubes (CNTs) is critical given the numerous expected applications of CNTs and their inevitable release into ecosystems. To explore the potential ecotoxicological effects of CNT surface modifications, we modified multi-walled carbon nanotubes (MWNTs) with polyethyleneimine (PEI) grafting, which endow the nanotubes with enhanced aqueous stability and, after additional chemical modifications, positive, negative, or neutral surface charges. Uptake and elimination experiments for PEI-MWNTs with various surface charges spiked to soils revealed the same limited earthworm accumulation and ready elimination as purified MWNTs. Interestingly, PEI-MWNTs and regular MWNTs revealed significantly different sorption and desorption behaviours in soils, which contrasts with their similar, limited bioaccumulation. Conversely, Daphnia magna, an aquatic organism, accumulated substantial concentrations of PEI MWNTs and was only able to excrete them with algae feeding. This suggests that the availability of food in ecosystems will substantially affect the long-term fate of nanotubes ingested by daphnia. Quantification of the carbon nanotubes in earthworms and daphnia was performed using 14C-labeled nanotubes. This approach overcomes significant measurement limitations to accurately determine CNT concentrations in environmentally relevant tissues and media. Additionally, PEI coatings increased nanotube toxicity to daphnia as exhibited by elevated percentages of immobilized daphnia after exposure to identical nanotube concentrations as regular MWNTs. Significant differences were observed among the various types of nanotubes suggesting that surface coatings may strongly influence the potential ecotoxicological effects of carbon nanotubes in the environment. 


\title{
SeParation of Single Wall Carbon Nanotubes (SWCNTS) USing FiEld-FloW FRACTIONATION TECHNIQUES
}

\author{
Frederick R. Phelan Jr. \\ National Institute of Standards and Technology, Polymers Division, \\ Gaithersburg, MD 20899-0854
}

The separation of single wall carbon nanotubes (SWCNTs) using Field-Flow Fractionation (FFF) techniques is discussed, with an emphasis on separation mechanism using different techniques. Results from both modeling and experiment show that traditional flow-FFF can be used to produce length based separations of SWCNTs controlled by the particle diffusion coefficient. Important differences between the separation mechanisms for spherical and rodlike particles are highlighted. Electric Field, Field-Flow Fractionation (EF-FFF) can be used to produce type separation (separation of metallic from semiconducting tubes) by exploiting both hydrodynamic properties and the polarizability differences between metallic and semi-conducting tubes. Differences between separation mechanisms for SWCNTs in both uniform and non-uniform electric fields are discussed. Sedimentation-FFF (Sd-FFF) produces separation based on density differences in an artificial gravitational field. Based on the development of a detailed understanding of SCWNT-surfactant complex transport properties, this technique potentially enables separation of SWCNTs by chirality. 